\title{
The influence of reducing fever on blood oxygen saturation in children
}

\author{
Shmuel Goldberg $^{1}$ (D) $\cdot$ Shmuel Heitner $^{2} \cdot$ Francis Mimouni $^{3} \cdot$ Leon Joseph $^{1}$ • \\ Reuben Bromiker ${ }^{3} \cdot$ Elie Picard $^{1}$
}

Received: 8 June 2017 /Revised: 24 October 2017 / Accepted: 25 October 2017 /Published online: 3 November 2017

(C) Springer-Verlag GmbH Germany 2017

\begin{abstract}
Laboratory-based studies on the oxyhemoglobin dissociation curve (ODC) suggest that high blood temperature decreases the affinity of hemoglobin for oxygen. The aim of the study was to evaluate the influence of pyrexia on oxygen saturation $\left(\mathrm{SpO}_{2}\right)$ in children presenting to the emergency department. Normoxemic children with body temperature at or above $38.5^{\circ} \mathrm{C}$ were included. Patients with a dynamic respiratory disease were excluded. $\mathrm{SpO}_{2}$ was measured before and after antipyretic treatment. The changes in body temperature and $\mathrm{SpO}_{2}$ were assessed and compared to the changes predicted from the ODC. Thirty-four children completed the study.
\end{abstract}

Communicated by Peter de Winter

Shmuel Goldberg

sgoldberg@szmc.org.il

Shmuel Heitner

shmirih@gmail.com

Francis Mimouni

fbmimouni@gmail.co

Leon Joseph

leonj@ doctors.org.uk

Reuben Bromiker

bromi59@gmail.com

Elie Picard

picard@szmc.org.il

1 Pediatric Pulmonology Institute, Shaare Zedek Medical Center, Hebrew University Medical School, P.O.B. 3235,

91301 Jerusalem, Israel

2 Pediatric Department, Shaare Zedek Medical Center, Hebrew University Medical School, Jerusalem, Israel

3 Neonatology Department, Shaare Zedek Medical Center, Hebrew University Medical School, Jerusalem, Israel
Mean temperature at presentation was $39.17 \pm 0.549{ }^{\circ} \mathrm{C}$ and mean $\mathrm{SpO}_{2}$ was $96.15 \pm 2.21 \%$. The mean decrease in temperature after antipyretic treatment was $1.71 \pm 0.67{ }^{\circ} \mathrm{C}$ and mean increase in $\mathrm{SpO}_{2}$ was $0.95 \pm 1.76 \%$. Among children in whom pyrexia decreased by $1.5^{\circ} \mathrm{C}$ or more, the mean increase in $\mathrm{SpO}_{2}$ was $1.45 \pm 1.57 \%$. The measured increase in $\mathrm{SpO}_{2}$ was close to the increase anticipated from the ODC.

Conclusion: Pyrexia was associated with decreased $\mathrm{SpO}_{2}$ in normoxemic children. The influence of pyrexia in children with low-normal oxygen saturation is expected to be much higher because of the non-linear shape of the ODC. Physicians treating patients with fever should be aware of this effect, especially in patients with borderline hypoxia.

What is Known:

- High blood temperature decreases the affinity of oxygen to hemoglobin.

- It is not known whether fever would decrease $\mathrm{SpO}_{2}$.

What is New:

- Fever is associated with decreased $\mathrm{SpO}_{2}$.

Keywords Fever · Oxygen saturation · Oxyhemoglobin dissociation curve
Abbreviations
ODC the oxyhemoglobin dissociation curve
$\mathrm{SaO}_{2}$ hemoglobin oxygen saturation
$\mathrm{SpO}_{2}$ oxygen saturation measured by pulse oximetry

\section{Introduction}

Non-invasive measurements of hemoglobin oxygen saturation $\left(\mathrm{SaO}_{2}\right)$ by pulse oximetry $\left(\mathrm{SpO}_{2}\right)$ have become routine in 
emergency settings, and $\mathrm{SpO}_{2}$ has been labeled the "fifth vital sign" [11]. Pulse oximetry is a measurement that reliably estimates $\mathrm{SaO}_{2}$ in arterial blood [6, 7, 9]. Single or serial measurements of $\mathrm{SpO}_{2}$ significantly influence decision-making in regard to workup, treatment, and hospitalization of patients. Many factors have been shown to influence $\mathrm{SaO}_{2}$ including: partial pressure of $\mathrm{O}_{2}$ and $\mathrm{CO}_{2}$ in blood, 2,3 DPG level, blood $\mathrm{pH}$, and temperature [2]. Increased blood temperature decreases the affinity of oxygen to hemoglobin, so that increasing blood temperature leads to a decrease in $\mathrm{SaO}_{2}$.

To the best of our knowledge, the effect of pyrexia upon $\mathrm{SpO}_{2}$ has not been quantified in children. Theoretically, pyrexia may affect the oxygen-hemoglobin dissociation curve directly, but it also may affect $\mathrm{SpO}_{2}$ through indirect mechanisms. For instance, pyrexia is associated with tachypnea which decreases $\mathrm{Pco}_{2}$ causing a shift of the oxygenhemoglobin dissociation curve and increasing $\mathrm{SpO}_{2}$. Additionally, pyrexia causes tachycardia and increased cardiac output which might shorten blood transit time in the lungs, leading to reduced oxygen uptake.

The aim of the current study was to evaluate the influence of pyrexia on $\mathrm{SpO}_{2}$ in a "real world" setting. For this purpose we measured $\mathrm{SpO}_{2}$ in febrile children presenting to the emergency room before and after antipyretic treatment. We compared our findings to those calculated from the oxygen dissociation curve.

\section{Methods}

Patients Children up to the age of 12 years old, who presented to the emergency department of our institution with pyrexia, were included in the study. Inclusion criteria were body temperature at or above $38.5^{\circ} \mathrm{C}$ and intention to use an antipyretic. Exclusion criteria were $\mathrm{SpO}_{2}$ below $90 \%$ and the presence of dynamic respiratory disease such as an asthma exacerbation, wheezing, bronchiolitis, or laryngitis. Patients with stable "respiratory disease" such as pneumonia without oxygen supplementation were included in the study.

Antipyretic treatment and vital signs measurements Oral acetaminophen $15 \mathrm{mg} / \mathrm{kg}$ or oral ibuprofen $10 \mathrm{mg} / \mathrm{kg}$ was given depending upon the preference of the ER physician. Before administration, we measured baseline vital signs including heart and respiratory rate, temperature, and oxygen saturation. Temperature was measured using a digital thermometer (Welch Allyn SureTemp® Plus 690 with a disposable tip) either orally or rectally, ensuring that the same method was used for all measurements for each child. Approximately $90 \mathrm{~min}$ after administration of antipyretics, vital signs including $\mathrm{SpO}_{2}$ and temperature were again measured.
Pulse oximetry measurements Pulse oximetry was measured using a Masimo Radical-7 motion resistant pulse oximeter (Masimo, Irvine, California, USA) incorporating Masimo SET v7.6.2.1 software. All measurements were conducted by the same investigator (S.H.). Measurements were conducted while the children were sleeping or awake and quiet (not feeding, fussy, or crying) before and after antipyretic therapy. Measurements were performed either on the palm of the hand or the foot (infants), or on a digit in older children. Both measurements for each child were performed at the same site. Using an averaging mode of $12 \mathrm{~s}$, the measurements were conducted for a period of at least $90 \mathrm{~s}$ after a stable and sharp pulsatile pulse waveform appeared and the perfusion index (PI) was steadily $>1$, all in accordance with the manufacturer's recommendations. We used trend data for analysis collected with Masimo Trendcom software provided by the manufacturer. $\mathrm{SpO}_{2}$, pulse rate, and perfusion index values (including alerts of low $\mathrm{SpO}_{2}$ signal quality) were recorded at 2 -s intervals. Trend data were omitted from analysis at least for the first $12 \mathrm{~s}$ of each recording and until $6 \mathrm{~s}$ of steady $\mathrm{SpO}_{2}$ recording was obtained. Any recordings that were missing a value or had a low $\mathrm{SpO}_{2}$ signal quality alert were also omitted from analysis. The average of all reliable $\mathrm{SpO}_{2}$ measurements was used.

Predicting the expected influence of blood temperature on $\mathrm{SpO}_{2}$ In order to calculate the expected influence of pyrexia on $\mathrm{SpO}_{2}$, we first calculated the expected alveolar $\mathrm{Po}_{2}\left(\mathrm{PAo}_{2}\right)$. For this purpose we used the alveolar gas equation: $\mathrm{PAO}_{2}=\mathrm{FiO}_{2}\left(\mathrm{~PB}-P_{\mathrm{H}_{2} \mathrm{O}}\right)-\mathrm{PAco}_{2}\left[\mathrm{FiO}_{2}+\left(1-\mathrm{FiO}_{2}\right) / R\right]$ (where $\mathrm{FiO}_{2}$ is the fractional inspired oxygen concentration in air, $\mathrm{PB}$ is the atmospheric (barometric) pressure, $P_{\mathrm{H} 20}$ is the partial pressure of water, and $\mathrm{PAco}_{2}$ is the partial $\mathrm{CO} 2$ alveolar pressure, while $R$ is the respiratory quotient [1]). Assuming $P_{\mathrm{H} 20}=47 \mathrm{mmHg}, \mathrm{PAco}_{2}=40 \mathrm{mmHg}$, and $R=0.8$, the $\mathrm{PAo}_{2}$ in a healthy examinee breathing room air at sea level (760 $\mathrm{mmHg}$ ) is expected to be $101.7 \mathrm{mmHg}$. The current study was done in Jerusalem, which is located at $800 \mathrm{~m}$ above sea level, where the barometric pressure is $697 \mathrm{mmHg}$ giving an anticipated $\mathrm{PAo}_{2}$ of $88.5 \mathrm{mmHg}$. Assuming that the alveolar-arterial gradient is $10 \mathrm{mmHg}$, the anticipated arterial $\mathrm{Po}_{2}\left(\mathrm{PaO}_{2}\right)$ is $91.7 \mathrm{mmHg}$ at sea level and $78.5 \mathrm{mmHg}$ in Jerusalem. In order to calculate the $\mathrm{SpO}_{2}$ from $\mathrm{PaO}_{2}$, we used an Internet-based calculator called "The Interactive Oxyhemoglobin Dissociation Curve" [8]. The software requires $\mathrm{PaO}_{2}, \mathrm{Pco}_{2}, \mathrm{pH}$, and temperature of the patient to calculate $\mathrm{SpO}_{2}$ and is based on the classic oxyhemoglobin dissociation curve equation developed by Kelman [3] and from data obtained by Severinghaus [10]. The calculations were all performed based upon the assumptions that $\mathrm{Pco}_{2}$ is $40 \mathrm{mmHg}$ and $\mathrm{pH}$ is at 7.38. The expected changes in $\mathrm{SpO}_{2}$ were calculated separately for each patient according to the temperature measured before and after antipyretic treatment. 
Table 1 Vital signs and oxygen saturation $($ mean \pm SD) before and after administration of antipyretic medication

\begin{tabular}{lllll}
\hline & Baseline & After antipyretics & Difference (before-after antipyretics) & $p$ value \\
\hline Temperature $\left({ }^{\circ} \mathrm{C}\right)$ & $39.17 \pm 0.54$ & $37.46 \pm 0.59$ & $1.71 \pm 0.67$ & $p<0.0001$ \\
Respiratory rate (breaths per minute) & $35.65 \pm 15.87$ & $28.97 \pm 12.54$ & $11.65 \pm 6.68$ & $p=0.002$ \\
Heart rate (beats per minute) & $156.33 \pm 21.8$ & $136.44 \pm 22.41$ & $19.9 \pm 14.5$ & $p=0.001$ \\
$\mathrm{SpO}_{2}$ & $96.15 \pm 2.13$ & $97.1 \pm 2.13$ & $-0.95 \pm 1.76$ & $p=0.0034$ \\
\hline
\end{tabular}

\section{Statistical analysis}

We used the Minitab 15 statistical software (Minitab, State College, Pennsylvania) for statistical analyses. We assessed the effect of fever reduction on $\mathrm{SpO}_{2}$ and other vital signs using paired $t$ tests. A $p$ value of $<0.05$ was considered significant.

\section{Results}

Forty-five patients were recruited for the study, and 34 (64.7\% males) completed the study protocol. The other 11 children did not complete the study protocol mostly because they were either crying or because they were not in the same state of arousal at the time of the second measurement. One patient was asleep during both measurements and all the others were awake at both measurements.

Twelve patients (35\%) were diagnosed with suspected viral infection, nine (26\%) with pneumonia, three $(9 \%)$ with acute gastroenteritis, three (9\%) with febrile seizures, and seven (21\%) with other diagnoses including tonsillitis, acute otitis media, familial Mediterranean fever, and lymphadenitis.

Mean patients' age was $37.5 \pm 14.8$ months, ranging 0.45 192 months, and mean weight was $14.2 \pm 10.5 \mathrm{~kg}$. Nineteen of the patients $(55.9 \%)$ were treated with acetaminophen and the rest (44.1\%) with ibuprofen.

Vital signs and oxygen saturation before and after administration of antipyretic agent are depicted in Table 1. In all participants, body temperature decreased, with a mean decrease of $1.71 \pm 0.67{ }^{\circ} \mathrm{C}(p<0.001)$. Both respiratory and heart rates decreased significantly. $\mathrm{SpO}_{2}$ increased significantly by $0.95 \pm 1.76 \%$ ( $p=0.0034)$. $\mathrm{SpO}_{2}$ increased in $73.5 \%$ $(25 / 34)$ of the whole cohort and in $90 \%(18 / 20)$ of children in whom body temperature decreased by $1.5{ }^{\circ} \mathrm{C}$ or more. There was a significant correlation between the decrease in temperature and the increase in $\mathrm{SpO}_{2}(p=0.031, R=0.34$, Fig. 1). The mean measured increase in $\mathrm{SpO}_{2}$ was only $56 \pm 114 \%$ of that expected when using Kelman's equation. Taking only patients $(n=20)$ in whom the temperature decreased by $\geq 1.5^{\circ} \mathrm{C}$, the measured increase in $\mathrm{SpO}_{2}$ was much closer to the anticipated increase $(90 \pm 105 \%$, Table 2$)$.

\section{Discussion}

In this study, we measured the $\mathrm{SpO}_{2}$ in febrile children in the emergency room and the influence of defervescence upon $\mathrm{SpO}_{2}$. We also compared our findings to expected changes based upon theoretical calculations using Kelman's equation. We found that most, but not all patients, had an increase in $\mathrm{SpO}_{2}$ concomitant with the decrease in body temperature. Moreover, a larger increase in $\mathrm{SpO}_{2}$ after defervescence correlated with a greater reduction in temperature. Finally, in patients in whom the decrease in temperature was greater (1.5 degrees and above), the mean increase in $\mathrm{SpO} 2$ was very close to the calculated theoretical increase.

To the best of our knowledge, there is only one study in the English written literature quantifying the influence of temperature upon $\mathrm{SpO}_{2}$ in patients with fever [4]. This study involved adults hospitalized in an intensive care unit and included patients receiving ventilator support and supplemental oxygen. An increased median temperature from 38.1 to $39.0^{\circ} \mathrm{C}$ was accompanied by a decrease in $\mathrm{SpO}_{2}$ from 98.0 to $97.6 \%$. Since the change in temperature was small and some (numbers are not given) of their patients were on ventilatory support, their findings might not be applicable to children breathing room air and with higher temperature changes. A previous study quantifying the influence of temperature upon $\mathrm{SpO}_{2}$ on 22 children was published by our group in Hebrew [5]. We determined the level of $\mathrm{SpO}_{2}$ by documenting the values as appeared on the pulse oximeter screen and not by averaging recorded values as was done in the current study. The results were similar to the current study. The mean decrease in

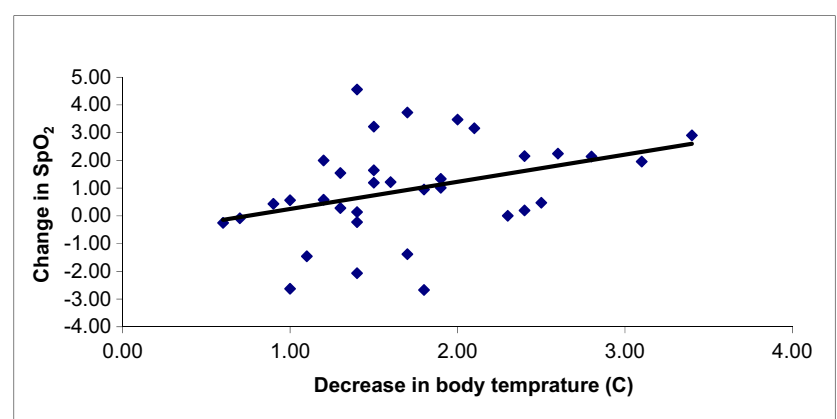

Fig. 1 The association between the change in body temperature and the change in $\mathrm{SpO}_{2}(p=0.031, R=0.34)$ 
Table 2 Comparison of the measured increase in $\mathrm{SpO}_{2}$ after antipyretic treatment to the predicted increase in $\mathrm{SpO}_{2}$. The comparison was performed on the whole cohort and then only on patients with a decrease in body temperature of $1.5^{\circ} \mathrm{C}$ or above

\begin{tabular}{llcccc}
\hline & & Temperature $\left({ }^{\circ} \mathrm{C}\right)$ & $\mathrm{Measured} \mathrm{SpO}_{2}(\%)$ & $\mathrm{Calculated} \mathrm{SpO}_{2}(\%)$ \\
\hline All participants $(n=34)$ & At baseline & $39.17 \pm 0.54$ & $96.15 \pm 2.21$ & $93.70 \pm 0.53$ \\
& After antipyretic administration & $37.46 \pm 0.59$ & $97.10 \pm 2.13$ & $95.08 \pm 0.4$ \\
& Difference & $-1.71 \pm 0.67$ & $0.95 \pm 1.76$ & $1.38 \pm 0.55$ \\
Only participants with temperature decrease of & At baseline & $39.34 \pm 0.58$ & $96.1 \pm 1.8$ & $93.53 \pm 0.57$ \\
$1.5^{\circ} \mathrm{C}$ or more $(n=20)$ & After antipyretic administration & $37.24 \pm 0.55$ & $97.6 \pm 1.6$ & $95.21 \pm 0.42$ \\
& Difference & $-2.125 \pm 0.55$ & $1.45 \pm 1.57$ & $1.68 \pm 0.47$ \\
\hline
\end{tabular}

temperature was $2.03{ }^{\circ} \mathrm{C}$ and the average rise in $\mathrm{SpO}_{2}$ was $1.55 \pm 1.79 \%(p=0.001)$.

In the current study, by definition, baseline $\mathrm{SpO}_{2}$ was above $90 \%$ in all patients. We speculate that the influence of an increase in body temperature on $\mathrm{SpO}_{2}$ in patients with low baseline $\mathrm{SpO}_{2}$ may be greater than that found in our study. This is because the oxyhemoglobin dissociation curve is much steeper when $\mathrm{Po}_{2}$ is lower than $60 \mathrm{mmHg}$. Indeed, according to Kelman's equation, when the $\mathrm{Po}_{2}$ is $60 \mathrm{mmHg}$ with a blood temperature of $37^{\circ} \mathrm{C}$, the anticipated $\mathrm{SpO}_{2}$ is $91 \%$. When the blood temperature is increased to $40{ }^{\circ} \mathrm{C}$, the anticipated $\mathrm{SpO}_{2}$ decreases to $85.8 \%$.

It is important to note that the calculation presented above is based upon the assumption that the values of $\mathrm{PAco}_{2}, R$ value, $P_{\mathrm{H} 20}$, barometric pressure, and the alveolar-arterial oxygen gradient are constant and equal to the values presented in the methods section. In practice, these values differ between patients and days. Moreover, the influence of compensatory mechanisms such as an increase in the respiratory rate is not known. Hence, the influence of an increased body temperature upon oxygen saturation will vary between patients and should be measured individually. We suggest that in a febrile patient, with borderline oxygen saturation, the decision to give supplemental $\mathrm{O}_{2}$, or to hospitalize, should be postponed until after the administration of antipyretics.

Study limitation The main limitation of the study is the absence of hypoxemic patients. It is anticipated that in those patients the influence of temperature upon $\mathrm{SpO}_{2}$ would be greater. We did not include them in the current study because those patients usually receive supplemental oxygen. Another study incorporating hypoxemic patient is required.

Conclusion Increased body temperature is associated with decreased $\mathrm{SpO}_{2}$ as suggested by the oxyhemoglobin dissociation curve. The relationship is not completely predictable. Physicians treating patients with fever should be aware of the potential significant influence of temperature on $\mathrm{SpO}_{2}$, especially in patients with borderline saturation. An increase in $\mathrm{SpO}_{2}$ in a patient after fever reduction should not be automatically interpreted as an improvement in the patients' respiratory condition. Similarly, a low $\mathrm{SpO}_{2}$ in a patient with high fever does not always imply significant respiratory compromise.

Acknowledgements We thank Masimo Corporation for the equipment support.

Authors' contributions Shmuel Goldberg: Dr. Goldberg conceptualized and design of the study, interpreted the results, reviewed and revised the manuscript, and wrote the final manuscript as submitted.

Shmuel Heitner: Dr. Heitner enrolled all participants, recorded the clinical and demographic data, performed all measurements, summarized the results, and approved the final manuscript as submitted.

Francis B. Mimouni: Prof. Mimouni helped conceptualizing and designing of the study, reviewed and revised the manuscript, and approved the final manuscript as submitted.

Leon Joseph: Dr. Joseph helped conceptualizing and designing of the study, reviewed and revised the manuscript, and approved the final manuscript as submitted.

Reuben Bromiker: Dr. Bromiker obtained the equipment support, critically reviewed the manuscript, and approved the final manuscript as submitted.

Elie Picard: Prof. Picard aided in the design of the study, initial analysis and interpretation of the results, critically reviewed the manuscript, and approved the final manuscript as submitted.

\section{Compliance with ethical standard}

Conflict of interest The authors declare that they have no conflict of interest.

Ethical approval All procedures performed in studies involving human participants were in accordance with the ethical standards of the institutional and national research committee and with the 1964 Helsinki Declaration and its later amendments or comparable ethical standards. The study was approved by the Shaare Zedek Medical Center Ethic Committee, study number 43/12.

Informed consent Written informed consent was obtained from all individual participants included in the study.

\section{References}

1. Grippi MA (1995) Pulmonary pathophysiology: Lippincott's pathophysiology series. Lippincott, Philadelphia, p 315

2. Guyton AC, Hall JE (2006) Transport of oxygen and carbon dioxide in blood and tissue fluids. In: Guyton AC, Hall JE (eds) 
Textbook of Medical Physiology, 11th edn. Elsevier Saunders, Philadelphia, pp 502-513

3. Kelman GR (1966) Digital computer subroutine for the conversion of oxygen tension into saturation. J Appl Physiol 21:1375-1376

4. Kiekkas P, Brokalaki H, Manolis E, Askotiri P, Karga M, Baltopoulos GI (2007) Fever and standard monitoring parameters of ICU patients: a descriptive study. Intensive Crit Care Nurs 23: 281-288

5. Lahav DZ, Picard E, Mimouni F, Joseph L, Goldberg S (2015) The effect of fever on blood oxygen saturation in children. Harefuah 154:162-165

6. Maneker AJ, Petrack EM, Krug SE (1995) Contribution of routine pulse oximetry to evaluation of patients with respiratory illness in a pediatric emergency department. Ann Emerg Med 25:36-40
7. Mower WR, Sachs C, Nicklin EL, Baraff LJ (1997) Pulse oximetry as a fifth pediatric vital sign. Pediatrics 99:681-686

8. Nielufar V (2000) The interactive oxyhemoglobin dissociation curve. Department of Medicine Medical College of Pennsylvania, East Falls http://www.ventworld.com/resources/oxydisso/ oxydisso.html. Last accessed 20/12/2015

9. Schnapp LM, Cohen NH (1990) Pulse oximetry: uses and abuses. Chest 98:1244-1250

10. Severinghaus JW (1979) Simple, accurate equations for human blood O2 dissociation computations. J Appl Physiol Respirat Environ Exercise Physiol 46:599-602

11. Tozzetti C, Adembri C, Modesti PA (2009) Pulse oximeter, the fifth vital sign: a safety belt or a prison of the mind? Intern Emerg Med 4:331-332 